\title{
NOTAS
}

\section{Una estructura funeraria Atavillos-Inca hallada en el sitio de Maxamarca, La Florida-Huaral}

\author{
AN ATAVILLOS-INCA FUNERAL STRUCTURE FOUND AT THE SITE OF \\ MAXAMARCA, LA FLORIDA-HUARAL
}

\author{
Pieter Dennis van Dalen Luna \\ http://orcid.org/oooo-0002-2498-9242 \\ Universidad Nacional Mayor de San Marcos \\ pvandalenl@unmsm.edu.pe \\ Noelia Allcca Aiquipa \\ http://orcid.org/0000-0003-4714-7766 \\ Universidad Nacional Mayor de San Marcos \\ noelia.allcca@unmsm.edu.pe
}

\author{
Marcos Morales Galarza \\ http://orcid.org/0000-0003-4854-3031 \\ Universidad Nacional Mayor de San Marcos \\ marco.morales7@unmsm.edu.pe \\ Yomira Silvia Huamán Santillán \\ http://orcid.org/oooo-0001-5209-9514 \\ Universidad Nacional Mayor de San Marcos \\ yomira.huaman@unmsm.edu.pe
}

\section{INTRODUCCIÓN}

La Comunidad Campesina de Pampas-La Florida, ubicado en el distrito de Atavillos Altos (provincia de Huaral) es ampliamente conocido por el complejo arqueológico de Rupac, que se encuentra ubicado en su jurisdicción, en la parte alta de la comunidad. Se trata del sitio arqueológico más conocido no solo de la cultura Atavillos, sino de toda la sierra del departamento de Lima. Se trata de un extenso asentamiento, con sectores como Marca Kullpi conformado por conjuntos arquitectónicos en los que cuatro o cinco de estos edificios se disponen alrededor de un patio central (Villar, 1935; Marussi, 1979; Cáceda, 2005; van Dalen, Grados, Medina y Malpartida, 2016; van Dalen, Grados, Medina, Malpartida y Tello, 2017; Grados, 2021). Rupac se ha

(C) Los autores. Este artículo es publicado por Arqueología y Sociedad del Museo de Arqueología y Antropología de la Universidad Nacional Mayor de San Marcos. Este es un artículo de acceso abierto, distribuido bajo los términos de la licencia Creative Commons Atribución 4.0 Internacional (CC BY 4.0) [https: / / creativecommons. org/licenses/by/4.0/deed.es] que permite el uso, distribución y reproducción en cualquier medio, siempre que la obra original sea debidamente citada de su fuente original. 
convertido en las últimas dos décadas en un sitio emblemático donde se da una fuerte afluencia turística en los fines de semana o feriados largos. Así, la Comunidad ha habilitado en el pueblo de La Florida hospedajes, restaurantes y otras instalaciones de recepción de visitantes.

Sin embargo, Rupac no es el único sitio arqueológico que existe en esta comunidad. Otro sitio que ha sido registrado arqueológicamente es Cerro Mango (Cáceda, 2014), cuyo nombre autóctono es Chuquimango (Duviols, 1967), conformado por un área ceremonial emplazada en la cima de esta montaña sagrada, las más importante de los Atavillos. Como parte del proyecto: "Determinación de las características socioculturales del patrimonio arqueológico de las comunidades campesinas altoandinas de la provincia de Huaral" (financiado por el VRIP de la Universidad Nacional mayor de San Marcos y de las comunidades y municipios involucrados) se logró registrar las características culturales de otros sitios arqueológicos ubicados en esta comunidad como: Caxamarca, Cuto, Canchapilca, Tambo Silencio, Buenavista, Jicarpuna, Acallca, Chicchipampa, Pippoma, cueva Huayopay, Corcopa, Mulacorral, Fortaleza de Huaroy, Supay Huanca, Curmac, entre muchos otros. Aunque la mayoría de estos sitios arqueológicos ubicados por encima y debajo del nivel del pueblo de La Florida pertenecen a la cultura Atavillos (periodos prehispánicos tardíos), hay también otros de mayor antigüedad, pertenecientes incluso al Precerámico. La gran densidad de sitios arqueológicos existente en esta comunidad evidencia la densa ocupación existente en su territorio desde periodos tempranos hasta la invasión hispana; con la presencia de numerosos pueblos que fueron reducidos hacia 1550 en el pueblo hispano de San Salvador de Pampas, pueblo que fue visitado hacia 1589 por el arzobispo de Lima Toribio de Mogrovejo (Benito, 2005), quien fuera más adelante canonizado como santo de la iglesia Católica.

\section{EL SITIO ARQUEOLÓGICO DE CAXAMARCA}

Se encuentra ubicado en la margen derecha del río Chacur, por debajo del nivel del pueblo de La Florida, en las coordenadas UTM: 303807 E y 8749479 N, a una altitud de $2388 \mathrm{~m} \mathrm{~s}$. n. m. Geográficamente, se ubica sobre un promontorio natural de dos cimas, a los cuales le han dado una mayor elevación cultural para la construcción de estructuras funerarias subterráneas. El sitio tiene una extensión aproximada de 3 hectáreas, sobre estas cimas que van descendiendo entre acantilados profundos y verticales. Está conformado por dos sectores:

Sector A: Ubicado sobre el promontorio más cercano al pueblo, muy destruido y alterado por el avance de la frontera agrícola y la construcción de un reservorio. Este sector se encuentra circundado por un muro perimétrico de $2 \mathrm{~m}$ de alto y $0.50 \mathrm{~m}$ de ancho, el cual encierra en su interior todas las edificaciones. En la cima misma se pueden aprecian los basamentos de edificaciones de planta cuadrangular y rectan- 
gular, con muros de 0.40 metros de grosor. En las laderas se aprecian los restos de estructuras funerarias de sección semisubterráneas. En la cima del promontorio se observan algunos habitáculos destruidos por remoción antrópica (acondicionamiento de andenes para siembra).

- Estructura 1: se encuentra sobre el promontorio. Tiene $2.20 \mathrm{~m}$ de largo y $2.10 \mathrm{~m}$ de ancho, es de planta cuadrangular. En el interior presenta piedras por colapso de los muros. Presenta restos de quema actual, y vegetación de tipo herbácea y cactácea.

- Estructura 2: Se observa solo el basamento de la estructura. Tiene $0.80 \mathrm{~m}$ de ancho, $1.00 \mathrm{~m}$ de largo y $0.80 \mathrm{~m}$ de profundidad aproximadamente. Se encuentra destruida.

- Estructura 3: Se observa la alineación de la estructura cuya construcción es a base de piedras canteadas unidas con argamasa y dispuestas en doble hilera. Tiene $1.00 \mathrm{~m}$ de largo y $0.90 \mathrm{~m}$ de ancho. Se observa además un muro que divide en dos secciones la estructura. En el interior se observa restos óseos.

Sector B: Se ubica en las coordenadas UTM: 303804E 8749446N a 2291 metros de altitud. Se ubica en la parte baja del asentamiento, sobre un promontorio natural debajo del sector $\mathrm{A}$. Al igual que el primer sector se encuentra circundado por un muro perimétrico, el cual es más visible en las coordenadas UTM: 301681E 8753440N (parte baja del asentamiento), donde se aprecia que está conformado por un muro de una sola hilera de $0.80 \mathrm{~m}$ de ancho, edificado con piedras de grandes dimensiones colocadas una sobre otra, sin presencia de argamasa y más de un metro de alto conservado. Este sector B se halla destruido casi en su totalidad, pues solo se logra apreciar algunas secciones de los muros de las edificaciones. En las coordenadas UTM: $303847 \mathrm{E}$ 8949362 se aprecia un muro que forma parte de una estructura destruida, elaborado a base de piedras canteadas sin argamasa, mampuesto uno sobre otro de manera vertical, presentando una sola hilera, se colocaron piedras de pequeñas dimensiones entre las piedras grandes con la finalidad de dar mayor estabilidad al muro. Este muro se encuentra en mal estado de conservación, presentando las siguientes dimensiones: $1.40 \mathrm{~m}$ de alto y $6 \mathrm{~m}$ de largo; presenta un nicho de $0.30 \mathrm{~m}$ de largo, $0.20 \mathrm{~m}$ de ancho y $0.20 \mathrm{~m}$ de profundidad, al interior del nicho se puede apreciar ofrendas realizadas por la población, la cual consiste en hojas de coca y una figurina de cerámica moderna.

En el mismo sector B en las coordenadas UTM: 303733 E 8749520 se ubica una estructura funeraria de planta cuadrangular, elaborada en base a piedras canteadas unidas con argamasa, presenta muros de una sola hilera. La estructura se encuentra en mal estado de conservación y cubierta de vegetación. Presenta las siguientes dimensiones: $1.80 \mathrm{~m}$ de largo, $1.60 \mathrm{~m}$ de ancho, con muros de $0.25 \mathrm{~m}$ de ancho y $1 \mathrm{~m}$ de altura; el vano de acceso se encuentra orientado al este, sin embargo, no se puede determinar las dimensiones del vano, debido al mal estado de conservación de la estructura. En las coordenadas UTM: 303753 E 8749506 se ubica otra estructura fu- 
neraria de planta cuadrangular, elaborada con piedras canteadas con argamasa, hallándose en mal estado de conservación (enterrada y destruida casi en su totalidad); presenta las siguientes dimensiones: $2 \mathrm{~m}$ de largo, $1.20 \mathrm{~m}$ de ancho y $1.10 \mathrm{~m}$ de altura. El vano de acceso se encuentra orientado al oeste; sin embargo, no se puede determinar las dimensiones del vano, debido al mal estado de conservación de la estructura.

En la parte superior del sitio se aprecian un conjunto de andenes cuyas graderías descienden hacia la ladera oeste del cerro, ubicado en las coordenadas UTM: 303871E $8749452 \mathrm{~N}$ y a 2360 metros de altitud. Presenta graderías de hasta $3.50 \mathrm{~m}$ de altura y un ancho de hasta 3 metros. Los muros de contención están edificados a base de piedra canteadas mampuestas sin argamasa.

En todo el sitio se registró material cultural asociado, como cerámica de estilos Quillahuaca, Inca local y Atavillos (local), de cocción mixta y acabado alisado, uno de los fragmentos presenta decoración. Además, se encontraron restos óseos humanos y de animales. Se encuentra en mal estado de conservación debido a las actividades antrópicas (agrícola), lo cual generó que la mayoría de los muros se encuentren colapsados y destruidos.

\section{LA ESTRUCTURA FUNERARIA ATAVILLOS-INCA}

En la ladera norte del promontorio que forma el sector A se identificó una estructura funeraria muy destruida en su cubierta y parte del paramento externo. Se trata de una estructura funeraria conformada por tres cámaras interconectadas internamente hacia el sureste. Esta estructura quedó expuesta debido a la remoción del terreno para ampliar el campo agrícola, se observa el talud. Presenta una cámara principal y dos cámaras secundarias, las cuales se encuentran construidas a base de piedras canteadas aplanadas unidas con argamasa y dispuestas en doble hilera. La cubierta de la estructura funeraria se encuentra construida con piedras grandes planas (lajas). El vano de acceso se encuentra orientado hacia el norte, el cual se encuentra destruido. La cámara principal tiene $2.00 \mathrm{~m}$ de largo, $1.60 \mathrm{~m}$ de ancho y $0.75 \mathrm{~m}$ de alto. Desde la cámara principal, se accede a dos cámaras secundarias:

- Cámara Secundaria 1: Tiene $1.20 \mathrm{~m}$ de largo, $0.60 \mathrm{~m}$ de ancho y $0.50 \mathrm{~m}$ de alto. El vano de acceso se orienta hacia el oeste, tiene $0.60 \mathrm{~m}$ de ancho, $0.50 \mathrm{~m}$ de alto y el muro $0.50 \mathrm{~m}$ de grosor.

- Cámara Secundaria 2: Tiene $1.20 \mathrm{~m}$ de largo, $0.60 \mathrm{~m}$ de ancho y $0.55 \mathrm{~m}$ de alto. El vano de acceso se orienta hacia el oeste y comunica con la cámara principal. El vano tiene $0.40 \mathrm{~m}$ de ancho y $0.52 \mathrm{~m}$ de alto.

En el interior de la estructura funeraria se identificó fragmentos de cerámica de una misma vasija, de estilo San Blas-Inca (del Tawantinsuyu), correspondiente a un aríbalo de altura conservada de $0.66 \mathrm{~m}$, aunque su altura era de aproximadamente 1 metro según sus atributos, diámetro de la boca de 13.2.cm; el borde evertido, con una 
aplicación en el cuerpo superior, asas laterales cintadas y verticales en el ecuador del cuerpo y presentando pintura roja en el cuerpo superior y naranja en el cuerpo inferior, siendo el cuello de color naranja. También se halló los restos de otra vasija Inca local (Atavillos-Inca), de base redondeada, borde evertido (aunque fragmentado), de $30 \mathrm{~cm}$ de alto y $7 \mathrm{~cm}$ de diámetro en la boca, pasta color naranja con algunas manchas de hollín por exposición al fuego. También se identificaron dos bordes de vasijas (cántaros) evertidos, lo que denota su filiación Inca local; así como un fragmento de estilo Quillahuaca (o Lauri Impreso) con dos círculos impresos. Así mismo se identificaron otros fragmentos de cerámica doméstica, llana, de pasta naranja. Se hallaron
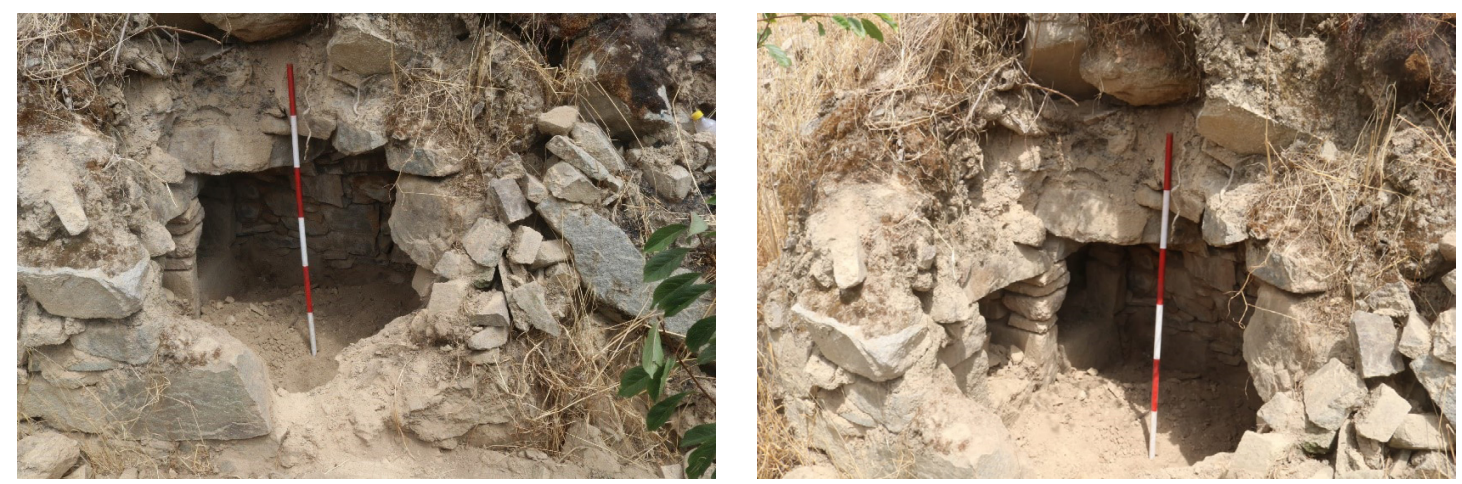

Figuras 1 y 2. Dos vistas de la estructura funeraria disturbada que contenía un contexto funerario Atavillos-Inca.
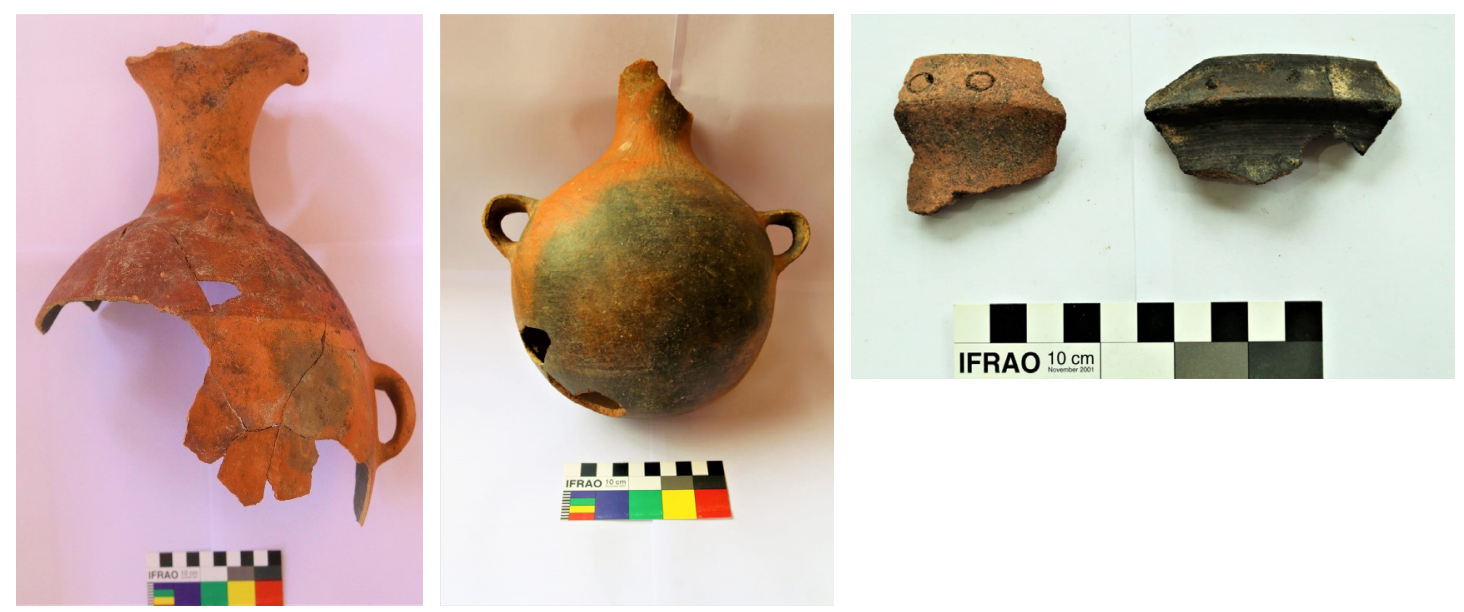

Figura 3 (izquierda). arybalo San Blas-Inca. Figura 4 (centro); vasija de borde evertido Atavillos-Inca. Figura 5 (derecha): fragmento cerámico de estilo Quillahuaca. 
restos botánicos, como corontas de maíz de tamaño pequeño, vainas de frijol seco, papas deshidratadas de tamaño pequeño (4 unidades) y semillas de algodón. Se halló restos óseos de cuy (huesos largos), vértebras de cánidos y huesos cortos de camélidos. No se observaron restos óseos humanos, salvo algunos huesos pequeños que no permiten determinar el sexo y edad del individuo, aunque al parecer se encuentran debajo de las piedras caídas producto de los escombros de los muros.

\section{RECONOCIMIENTOS}

Las investigaciones en la Comunidad Campesina de Pampas - La Florida fueron realizadas gracias al apoyo de la Universidad Nacional Mayor de San Marcos través del Vicerrectorado de Investigación y Posgrado; así como de la Comunidad Campesina de Pampas. El registro del sitio de Caxamarca se realizó en compañía de ellos, estudiantes de arqueología de la UNMSM, Fabiola Kupa Acuña y José Vasquez Marín; así como los guías y comuneros Leoncio Rojas Salas, Aníbal Celedonio Soto y José Rojas Morales.

\section{CONCLUSIONES}

Las labores de prospección arqueológica desarrollados en la Comunidad Campesina de San Salvador de Pampas permitieron la ubicación de una estructura funeraria disturbada en el sitio de Caxamarca, ubicado por debajo y a pocos metros del pueblo de La Florida. Se trata de una estructura de tres cámaras subterráneas (una principal y dos secundarias) ubicadas en el sector A, en cuyo interior se apreció como parte de los materiales asociados disturbados cerámica Inca local, con sus características propias como los bordes evertidos. Por ello concluimos que este contexto funerario corresponde al Tawantinsuyu, conformado por un contexto Atavillos - Inca; es decir, de un personaje local que tuvo un cargo como parte de la estructura orgánica del Estado Inca en territorio Atavillos.

\section{REFERENCIAS BIBLIOGRÁFICAS}

Benito, J. A. (2005). Libro de visitas de Santo Toribio de Mogrovejo (1593-1605). Lima: Pontificia Universidad Católica del Perú.

Cáceda, D. (2005). Determinación arqueológica de las características culturales en los sitios de Rúpac, Chiprac, Añay y Cerro mango. Revista Cultural Kullpi. Investigaciones culturales en la provincia de Huaral y el norte chico, 2, pp. 23-56. Huaral.

Cáceda, D. (2014). Cerro Mango Cápac: Un apu Atavillos en la cuenca alta del río Chancay. En: P. van Dalen, (ed.). Arqueología de las cuencas alto y medio andinas del departamento de Lima (pp. 321-326). Lima. 
Duviols, P. (1967). Un inédit de Cristobal de Albornoz: La instrucción para descubrir todas las guacas del Pirú y sus camayos y haciendas. Journal de la société des américanistes, 56(1), 7-39.

Grados, H. (2021). Función y patrón arquitectónico de los edificios tipo Kullpi del sector A del sitio arqueológico de Rupac, Atavillos bajo, Huaral. Tesis de licenciatura para optar el título profesional en arqueología. Lima: Universidad Nacional Mayor de San Marcos.

Marussi Castellán, Ferrucho. (1979). (1979) Rupac: análisis urbanístico de una ciudad prehispánica. Bulletin de l'Institut Francais d'Etudes Andines, VIII (1, 2, 3). Lima.

van Dalen Luna, P., Grados, H., Medina, F., y Malpartida, M (2016). Conviviendo con los ancestros: investigaciones arqueológicas en Rúpac, Huaral. Arqueología y Sociedad 30, 425-472. Lima: Universidad Nacional Mayor de San Marcos.

van Dalen Luna, P., Grados, H., Medina, F., Tello, R. y Malpartida, M. (2017). Arqueología de Rúpac, un sitio Atavillos en la cuenca alta del río Chancay - Huaral. Actas del II Congreso Nacional de Arqueología (pp. 179-188). Lima: Ministerio de Cultura.

Villar Córdova, P. (1935). Arqueología del departamento de Lima. Lima.

\section{SOBRE LOS AUTORES}

\section{Pieter Dennis van Dalen Luna}

Licenciado en Arqueología (UNMSM), bachiller en Ciencias de la Educación (UNEEGV-LC), magíster en Arqueología Andina (UNMSM), magíster en Gestión del Patrimonio Cultural (UNMSM). Doctor en Ciencias Sociales con mención en antropología (UNMSM) y Doctorado en el Programa de Estudios Andinos Arqueología especialidad en arqueología (PUCP). Diplomado en Conservación especializado en arquitectura arqueológica. Docente nombrado de la UNMSM, departamento académico de arqueología. Premio al Mérito Científico UNMSM 2012. Exdirector del Museo de Arqueología y Antropología de San Marcos-UNMSM (2012-2017). Exvicedecano nacional del Colegio de Arqueólogos (2018-2019). Director del proyecto de Investigación Arqueológica Chancay-Huaral-Atavillos (PACHA). 


\section{Noelia Allcca Aiquipa}

Inició sus estudios superiores en la E. A. P. de Arqueología de la Universidad Nacional Mayor de San Marcos en el año 2016 y egresó en febrero del año 2021.

En el año 2020 durante sus estudios en la UNMSM, participó y ganó en el concurso de financiamiento del VRIP, con su trabajo de investigación titulado "Palacio de Oquendo, una residencia de élite en el valle bajo del río Chillón" .

Su interés académico es investigar periodos tardíos: Intermedio Tardío y Horizonte Tardío. Actualmente investiga la zona baja del valle del río Chillón.

\section{Marco Antonio Morales Galarza}

Estudiante de último ciclo de la carrera de arqueología de la Universidad Nacional Mayor de San Marcos, interesado en realizar investigaciones sobre arqueología del paisaje y patrones de asentamiento de las sociedades prehispánicas tardías. Actualmente viene desarrollando investigaciones arqueológicas en la sub cuenca del río Lampián, cuenca alta del río Chancay-Huaral.

\section{Yomira Silvia Huamán Santillán}

Egresada en Arqueología en la Universidad Nacional Mayor de San Marcos. Dirigió la investigación: "Cajamarquilla, identificación de su máxima extensión a través de la arquitectura del tapial en el Periodo Intermedio Tardío" (E2015007C), proyecto ganador del Programa de Promoción de trabajo de investigación para optar el grado de Bachiller, del Vicerrectorado de Investigación y Posgrado de la Universidad Nacional Mayor de San Marcos. Se desempeñó como arqueóloga de campo y gabinete en el "Proyecto Arqueológico La Huaca". Actualmente se desempeña como jefe de campo en el proyecto "Determinación de las características socioculturales del patrimonio arqueológicos de las comunidades campesinas altoandinas de la provincia de Huaral." Y también como responsable del "Proyecto arqueológico con escuela de campo, Cajamarquilla $2021^{\prime \prime}$. 